\title{
ERRATUM
}

Andreas Perrot - Hajo Schmidt-Traub - Bernard Hoffmann - Matthias Prager • Nana Bit-Avragim - Raisa I. Rudenko - Dinara A. Usupbaeva - Zhyldyz Kabaeva • Bakytbek Imanov • Mirsaid M. Mirrakhimov • Heiko Witt • Rainer Dietz • Anna Wycisk - Michal Tendera · Reinhard Geßner · Karl Josef Osterziel

\section{Prevalence of cardiac beta-myosin heavy chain gene mutations in patients with hypertrophic cardiomyopathy}

Published online: 22 September 2005

(C) Springer-Verlag 2005

\section{J Mol Med (2005) 83:468-477}

Heiko Witt should have been included as the eleventh author of this paper. His affiliation is Charité-Universitätsmedizin Berlin, Institut für Laboratoriumsmedizin und Pathobiochemie, Campus Virchow-Klinikum, Germany.

The online version of the original article can be found at http://dx.doi.org/10.1007/s00109-005-0635-7

A. Perrot $(\bowtie) \cdot$ N. Bit-Avragim $\cdot$ Z. Kabaeva $\cdot$ R. Dietz

K. J. Osterziel

Kardiologie am Campus Buch und Virchow-Klinikum,

Charité-Universitätsmedizin Berlin und Max-Delbrück-Centrum

für Molekulare Medizin,

Wiltbergstrasse 50,

13125 Berlin, Germany

e-mail: perrot $@$ fvk-berlin.de

Tel.: +49-30-94172508

Fax: +49-30-94172279

H. Schmidt-Traub · B. Hoffmann · M. Prager - H. Witt •

R. Geßner

Institut für Laboratoriumsmedizin und Pathobiochemie am

Campus Virchow-Klinikum, Charité-Universitätsmedizin,

Berlin, Germany

R. I. Rudenko - D. A. Usupbaeva · B. Imanov ·

M. M. Mirrakhimov

National Center for Cardiology and Internal Medicine,

Bishkek, Kyrgyzstan

A. Wycisk $\cdot$ M. Tendera

Third Department of Cardiology, Silesian School of Medicine,

Katowice, Poland 